Notre Dame Journal of Formal Logic

Volume VII, Number 3, July 1966

\title{
ON MODAL RENDERINGS OF INTUITIONISTIC PROPOSITIONAL LOGIC
}

\section{NICHOLAS RESCHER}

The intuitionistic propositional calculus (IPC) of Heyting ${ }^{1}$ rests upon the following eleven axioms:
(A1) $p \rightarrow(p \wedge p)$
(A2) $(p \wedge q) \rightarrow(q \wedge p)$
(A3) $(p \rightarrow q) \rightarrow[(p \wedge r) \rightarrow(q \wedge \gamma)]$
(A4) $[(p \rightarrow q) \wedge(p \rightarrow r)] \rightarrow[p \rightarrow r]$
(A5) $q \rightarrow(p \rightarrow q)$
(A6) $[p \wedge(p \rightarrow q)] \rightarrow q$
(A7) $p \rightarrow(p \underline{v} q)$
(A8) $(p \underline{\vee} q) \rightarrow(q \underline{v} p)$
(A9) $[(p \rightarrow r) \wedge(q \rightarrow \gamma)] \rightarrow[(p \underline{\vee} q) \rightarrow r]$
$(\mathrm{A} 10) \neg p \rightarrow(p \rightarrow q)$
(A11) $[(p \rightarrow q) \wedge(p \rightarrow \neg q)] \rightarrow \neg p$

Here the symbols ' $\rightarrow$ ', ' $\wedge$ ', ' $\underline{\vee}$ ', and ' $\neg$ ' are used for intuitionistic implication, conjunction, disjunction, and negation, respectively.

Moreover, there are certain theses which Heyting in his book of 1956 specifically and explicitly rejects as intuitionistically unacceptable:

(U1) $(p \vee q) \rightarrow(p \vee q)$ [See p. 97.]

(U2) $\sim p \rightarrow \neg p$ [See pp. 18-19, 97-98.]

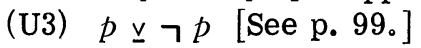

(U4) ᄀᄀ $p \rightarrow p$ [See p. 99.]

(U5) $(p \rightarrow q) \underline{v}(q \rightarrow p) \quad$ [See p. 99.]

(U6) $\neg(p \wedge q) \rightarrow(\neg p \vee \neg q)$ [See p. 100.]

(U7) $(\neg q \rightarrow \neg p) \rightarrow(p \rightarrow q)$ [See p. 101.]

(U8) $\neg\urcorner(p \underline{\vee} q) \rightarrow(\neg \neg p \underline{v} \neg \neg q)$ [See p. 101.]

The symbols ' $\sim$ ', ' $\&$ ', and ' $v$ ' will be used for "ordinary" (non-intuitionistic) negation, conjunction, and disjunction, respectively; and ' $\supset$ ' will be used below for material implication.

Various "dictionaries" for "translating" statement schemata of IPC into the vocabulary of Lewis' systems of strict implication have been or can 
be discussed. We shall consider the five following possibilities (" $N$ " represents the modality of necessity, " 3 " the relation of strict implication). ${ }^{2}$

IPC-vocabulary

Lewis-vocabulary

\begin{tabular}{l|r|r|r|r|r} 
& \multicolumn{1}{|c|}{$D 1$} & $D 2$ & \multicolumn{1}{c|}{$D 3$} & \multicolumn{1}{c|}{$D 4$} & \multicolumn{1}{c|}{$D 5$} \\
\hline$a$ (variable) & -- & -- & $N a$ & $a$ & $a$ \\
$S$ (arbitrary schema) & $S$ & $S$ & -- & -- & -- \\
$\neg \quad S$ & $\sim N S$ & $N \sim S$ & $N \sim S$ & $\sim N S$ & $N \sim N S$ \\
$S \rightarrow T$ & $N S-N T$ & $S-T$ & $S-T$ & $N S \supset N T$ & $N S \supset N T$ \\
$S \underline{\vee} T$ & $N S \vee N T$ & $N S \vee N T$ & $S \vee T$ & $N S \vee N T$ & $N S \vee N T$ \\
$S \wedge T$ & $S \& T$ & $N S \& N T$ & $S \& T$ & $S \& T$ & $N S \& N T$
\end{tabular}

Dictionary D1 was discussed by Kurt Gödel in his important paper of 1931, and dictionaries D3-D5 were discussed by McKinsey and Tarski in a paper of 1948 that extended Gödel's findings. Dictionary D2 is, so far as I know, novel, but is patently closely akin to D1 on the one hand, and D3 on the other.

The result established by Gödel is this, that if we take Lewis' system S4 as governing the modal concepts, then dictionary D1 translates all IPCtheorems into S4 theorems and all IPC non-theorems into non-theorems of S4. This result was extended with respect to dictionaries D3-D5 by McKinsey and Tarski.

These results, however, envisage only the translation of formulas expressed in purely intuitionistic symbolism. They do not apply when one introduces "mixed" formulas in which both intuitionistic and classical connectives occur, such as the intuitionistically rejected (U1) and (U2). (It is supposed that in "translating" such mixed formulas all the nonintuitionistic connectives are unaffected and remain unchanged. ${ }^{3}$ ) Indeed, D1 translates (U2) into a theorem of S4, and so do D4 and D5. D3 on the other hand translates (U1) into a theorem of S4.

The advantage of D2 vis a vis the other "dictionaries" is that it translates all of the U-theses into non-theorems of S4. A seeming disadvantage is that it translates IPC axioms (A5) and (A7) into non-theorems of S4. But this disadvantage is not so serious as it at first seems. For if these two axioms were replaced by

$\left(\mathrm{A}^{\prime} 5\right) \mathrm{Nq} \rightarrow(p \rightarrow q)$

$\left(\mathrm{A}^{\prime} 7\right) N p \rightarrow(p \underline{q} q)$

or by some equivalent formulation using purely intuitionistic vocabulary, then they too would go over into theorems of S4. I am not certain that such a revision of (A5) and (A7) would be acceptable to intuitionists. But I can think only of considerations that would make it palatable. After all, the principal function of an intuitionistic thesis of the form "... $\rightarrow--$ " is, as Heyting's discussion makes clear, to inform us as to the inferences that can be made once "..." is established (i.e., is "necessary" in some intuitionistically appropriate sense). And thus the added " $N$ " in the antecedents of $\left(A^{\prime} 5\right)$ and $\left(A^{\prime} 7\right)$ seems entirely commensurate with intuitionistic objectives. 
It is also germane to recall the criticism of theses akin to the original (A5) as being "paradoxes" of implication."

It is worthwhile to raise the question of the consequences of strengthening the underlying modal system from S4 to S5. If, following Gödel's lead, we construe the $\mathrm{N}$-modality as representing provability (being "beweisbar"), we should expect that something should "go wrong" when this step is taken, in the sense that some of the unacceptable U-theses should now become theorems. (For the characteristic axiom of S5, viz. " $\sim N \sim N p-3 \mathrm{~Np}$ " is clearly false under this construction-the fact that " $p$ " is not provably unprovable does not assure the provability of " $p$ ".) This expectation is met. For example, with all of the "dictionaries," the transform of (U8) is a non-theorem of S4 that becomes a theorem in S5 (and if dictionary D2 is put aside, this is also the case with (U6) and (U7)).

The data on which our discussion has been based are summarized in the following tabulation: ${ }^{5}$

THE STATUS OF THE MODAL "TRANSLATIONS"

OF INTUITIONISTIC THESE

\begin{tabular}{cccccc} 
& $\mathrm{D} 1$ & $\mathrm{D} 2$ & $\mathrm{D} 3$ & $\mathrm{D} 4$ & $\mathrm{D} 5$ \\
\hline Axioms $^{6}$ & & & & & \\
$(\mathrm{~A} 1)$ & $\mathbf{T}$ & $\mathbf{T}$ & $\mathbf{T}$ & $\mathbf{T}$ & $\mathbf{T}$ \\
$(\mathrm{A} 2)$ & $\mathbf{T}$ & $\mathbf{T}$ & $\mathbf{T}$ & $\mathbf{T}$ & $\mathbf{T}$ \\
$(\mathrm{A} 3)$ & $\mathbf{T}$ & $\mathbf{T}$ & $\mathbf{T}$ & $\mathbf{T}$ & $\mathbf{T}$ \\
$(\mathrm{A} 4)$ & $\mathbf{T}$ & $\mathbf{T}$ & $\mathbf{T}$ & $\mathbf{T}$ & $\mathbf{T}$ \\
$(\mathrm{A} 5)$ & $\mathbf{T}$ & $\mathbf{N}$ & $\mathbf{T}$ & $\mathbf{T}$ & $\mathbf{T}$ \\
$(\mathrm{A})$ & $\mathbf{T}$ & $\mathbf{T}$ & $\mathbf{T}$ & $\mathbf{T}$ & $\mathbf{T}$ \\
$(\mathrm{A} 7)$ & $\mathbf{T}$ & $\mathbf{T}$ & $\mathbf{T}$ & $\mathbf{T}$ & $\mathbf{T}$ \\
$\left(\mathrm{A}^{\prime} 7\right)$ & $\mathbf{T}$ & $\mathbf{T}$ & $\mathbf{T}$ & $\mathbf{T}$ & $\mathbf{T}$ \\
$(\mathrm{A} 8)$ & $\mathbf{T}$ & $\mathbf{T}$ & $\mathbf{T}$ & $\mathbf{T}$ & $\mathbf{T}$ \\
$(\mathrm{A} 10)$ & $\mathbf{T}$ & $\mathbf{T}$ & $\mathbf{T}$ & $\mathbf{T}$ & $\mathbf{T}$ \\
$(\mathrm{A} 11)$ & $\mathbf{T}$ & $\mathbf{T}$ & $\mathbf{T}$ & $\mathbf{T}$ & $\mathbf{T}$ \\
\end{tabular}

Rejected Theses

$\begin{array}{llllll}\text { (U1) } & \mathbf{N} & \mathbf{N} & \mathbf{T} & \mathbf{N} & \mathbf{N} \\ \text { (U2) } & \mathbf{T} & \mathbf{N} & \mathbf{N}^{*} & \mathbf{T} & \mathbf{T} \\ \text { (U3) } & \mathbf{N}^{*} & \mathbf{N} & \mathbf{N} & \mathbf{N}^{*} & \mathbf{N}^{*} \\ \text { (U4) } & \mathbf{N}^{*} & \mathbf{N} & \mathbf{N}^{*} & \mathbf{N}^{*} & \mathbf{N} \\ \text { (U5) } & \mathbf{N} & \mathbf{N} & \mathbf{N} & \mathbf{N} & \mathbf{N} \\ \text { (U6) } & \mathbf{N}^{*} & \mathbf{N} & \mathbf{N}^{*} & \mathbf{N}^{*} & \mathbf{N}^{*} \\ \text { (U7) } & \mathbf{N}^{*} & \mathbf{N} & \mathbf{N}^{*} & \mathbf{N}^{*} & \mathbf{N}^{*} \\ \text { (U8) } & \mathbf{N}^{*} & \mathbf{N}^{*} & \mathbf{N}^{*} & \mathbf{N}^{*} & \mathbf{N}^{*}\end{array}$

Key $\quad \mathrm{T}=$ theorem of $\mathrm{S} 4$ (and S5)

$\mathrm{N}=$ non-theorem of $\mathrm{S} 4$ and $\mathrm{S} 5$

$\mathrm{N}^{*}=$ non-theorem of $\mathrm{S} 4$ that becomes a theorem of S5 


\section{NOTES}

1. Based on axioms first presented in Heyting [1], but now more readily accesstble in Heyting [5], see p. 101.

2. It is assumed throughout this paper that the only translational changes are those specif ied by the "dictionaries" under consideration.

3. This procedure is adopted to assure that all of the standard, non-intuitionistic theses of the initial, mixed system go over into theorems. Certain trivial, pointless departures from this policy are possible by making use of standard equivalences (e.g., by such a rule as, "Translate ' $\sim S$ ' as ' $\sim \sim S$ ") - but the introduction of any modal connectives must be eschewed.

4. However, if the proposed change of axioms (A5) and (A7) were adopted, it would be necessary to augment the resulting set of axioms somewhat if the usual set of "intuitionistically desirable" theses is to be forthcoming as theorems. For, as Heyting observes (p. 101 of reference [5]), it is desired to have

$(\neg(p \wedge q) \wedge p) \rightarrow \neg q$

as well as its logical consequence

$(\neg า p \wedge \neg า q) \rightarrow \neg า(p \wedge q)$

as intuitionistically acceptable theses. But it is readily shown that these are independent of the revised axiom-set. For if these theses are translated into modal vocabulary by our last-given "dictionary" they-unlike the IPC axioms-go over into non-theorems of S5-as is readily seen by the use of Lewis' "Group III."

5. I wish to thank Miss Barbara Anne Hunt for her help in checking the data compiled in this tabulation.

6. The validity of the rules of inference (viz. modus ponens for implication and the intersubstitutability of mutual implicants) is of course also preserved by these translationschemes.

\section{REFERENCES}

[1] A. Heyting. "Die formalen Regeln der intuitionistischen Logik." Sitzungsberichte der Preussischen Akademie der Wissenschaften (Physikalisch-mathematische Klasse), 1930, pp. 42-56.

[2] C. I. Lewis and C. H. Langford. Symbolic Logic. New York, 1932; second edition, New York, 1959.

[3] Kurt Gödel. "Eine Interpretation des intuitionistischen Aussagen-kalküls." Ergebnisse eines mathematischen Kolloquiums, vol. 4 (1933), pp. 39-40. Reporting results presented in 1931.

[4] J. J. C. McKinsey and Alfred Tarski. "Some Theorems About the Sentential Calculi of Lewis and Heyting." The Journal of Symbolic Logic, vol. 13 (1948), pp. 1-15.

[5] A. Heyting. Intuitionism. Amsterdam, 1956.

University of Pittsburgh

Pittsburgh, Pennsylvania 